\title{
INTRAVESICAL CAPSAICIN VERSUS RESINIFERATOXIN IN PATIENTS WITH DETRUSOR HYPERREFLEXIA: A PROSPECTIVE RANDOMIZED STUDY
}

\author{
ANTONELLA GIANNANTONI,* SAVINO M. DI STASI, ROBERT L. STEPHEN, PIERLUIGI NAVARRA, \\ GIORGIO SCIVOLETTO, ETTORE MEARINI AND MASSIMO PORENA
}

From the Department of Urology, University of Perugia, Perugia, Department of Urology "Tor Vergata," University of Rome, Institute of Pharmacology Catholic University of Rome and IRCCS S. Lucia Rehabilitation Hospital, Rome and Physion Laboratories, Medolla, Italy

\section{ABSTRACT}

Purpose: Capsaicin and resiniferatoxin (Sigma Chemical Co., St. Louis, Missouri) administered intravesically are attractive options for treating detrusor hyperreflexia. Because the 2 agents differ in chemical structure and relative potency, possible differences in their clinical and urodynamic effects were investigated in this prospective comparative study.

Materials and Methods: A group of 24 spinal cord injured patients with refractory detrusor hyperreflexia were randomly assigned to receive a single dose of $2 \mathrm{mM}$. capsaicin in $30 \mathrm{ml}$. ethanol plus $70 \mathrm{ml} .0 .9 \%$ sodium chloride or $100 \mathrm{nM}$. resiniferatoxin in $100 \mathrm{ml} .0 .9 \%$ sodium chloride. Dwell time was 40 minutes with urodynamic monitoring. Urodynamics were performed at baseline before treatment, and after followups of 30 and 60 days. The frequency of daily catheterizations, incontinence episodes and side effects was recorded.

Results: There was no significant urodynamic or clinical improvement in the capsaicin arm at 30 and 60 days of followup. In the resiniferatoxin arm the mean uninhibited detrusor contraction threshold plus or minus standard deviation increased from $176 \pm 54$ to $250 \pm 107 \mathrm{ml}$. at 30 days $(\mathrm{p}<0.05)$ and to $275 \pm 98 \mathrm{ml}$. at 60 days $(\mathrm{p}<0.01)$. Mean maximum bladder capacity increased from $196 \pm 75$ to $365 \pm 113 \mathrm{ml}$. at 30 days $(\mathrm{p}<0.001)$ and to $357 \pm 101 \mathrm{ml}$. at 60 days $(\mathrm{p}<0.001)$. Daily catheterizations and incontinent episodes were significantly decreased at 30 and 60 days of followup. Autonomic dysreflexia, limb spasms, suprapubic discomfort and hematuria developed in most patients who received capsaicin but in none who received resiniferatoxin.

Conclusions: Intravesical administration of resiniferatoxin is superior to that of capsaicin in terms of urodynamic results and clinical benefits in spinal cord injured patients and it does not cause the inflammatory side effects associated with capsaicin.

KEY WoRDS: bladder, capsaicin, spinal cord injuries, urinary incontinence

Current strategies for treating detrusor hyperreflexia due to neurological disease rely on partial blockade of parasympathetic innervation to the bladder using oral anticholinergic drugs. Although they are successful in most cases, anticholinergics such as oxybutynin have disagreeable side effects that cause a proportion of patients to abandon them at doses insufficient to restore continence. ${ }^{1}$ Some patients respond to intravesical oxybutynin but no approved formulation is available for this method of administration. Furthermore, some patients do not respond to either treatment.

Inhibiting transmission from the bladder along the afferent C-fibers by intravesical administration of compounds collectively described as vanilloids is an attractive option. Capsaicin was the first such agent used and it achieves durable, months-long beneficial effects in about $60 \%$ to $70 \%$ of patients. ${ }^{2}$ However, topical administration is immediately followed by bladder irritation, an effect ascribed to initial stimulation before C-fibers inactivation. Resiniferatoxin, which is about 1,000 -fold more potent than capsaicin, also acts on vanilloid receptors. ${ }^{2}$ Intravesical administration of this agent causes prolonged inactivation of $\mathrm{C}$-fibers yet with minimal and/or absent initial stimulatory effects. ${ }^{3}$ Capsaicin and resiniferatoxin differ in their chemical structures and relative potency, so that there may be substantial differences in clinical and urodynamic effects. We investigated the effectiveness and safety of capsaicin and resiniferatoxin intraves-

Accepted for publication November 2, 2001.

* Requests for reprints: Via Torrice n. 4, 00189 Rome, Italy. ical administration, comparing their clinical and urodynamic effects in a group of spinal cord injured patients with detrusor hyperreflexia unresponsive to conventional anticholinergic therapy.

\section{MATERIALS AND METHODS}

Patient characteristics. Enrolled in this study were 16 male and 8 female chronic spinal cord injured patients. Table 1 lists clinical features and American Spinal Injury Association impairment. ${ }^{4}$ The study was approved by the ethics committee. All patients were informed about the scientific nature of the study and provided written consent.

Preliminary assessment. Standard evaluation included a history, physical examination, serum chemistries, urinalysis, urine culture and imaging assessment of urinary tract by ultrasound, excretory urography and cystourethrography. Baseline urodynamic evaluation followed International Continence Society standards ${ }^{5}$ and was performed 1 month before commencing the study. Maximum bladder capacity and bladder compliance, uninhibited detrusor contraction threshold and maximum amplitude, and urinary leakage were recorded. Study inclusion criteria were unacceptable clinical and urodynamic detrusor activity suppression by oral and intravesical oxybutynin, intolerable anticholinergic side effects, normal renal function, no vesicoureteral reflux or abnormal upper urinary tracts. All patients performed clean intermittent catheterization and continued on $5 \mathrm{mg}$. oxybu- 
TABLE 1. Patient characteristics

\begin{tabular}{|c|c|c|c|}
\hline $\begin{array}{l}\text { Pt. - Sex - Age } \\
\text { No. }\end{array}$ & $\begin{array}{l}\text { Lesion } \\
\text { Level }\end{array}$ & $\begin{array}{l}\text { American } \\
\text { Spinal Injury } \\
\text { Association } \\
\text { Impairment }\end{array}$ & $\begin{array}{c}\text { Disease } \\
\text { Duration } \\
\text { (mos.) }\end{array}$ \\
\hline & \multicolumn{3}{|c|}{ Capsaicin group } \\
\hline $1-F-32$ & $\mathrm{C} 7$ & B & 49 \\
\hline $2-\mathrm{M}-42$ & $\mathrm{~T} 8$ & B & 51 \\
\hline $3-\mathrm{M}-33$ & $\mathrm{~T} 10$ & $\mathrm{~B}$ & 48 \\
\hline $4-\mathrm{M}-34$ & $\mathrm{~T} 6$ & $\mathrm{~A}$ & 50 \\
\hline $5-F-36$ & $\mathrm{~T} 10$ & $\mathrm{~B}$ & 58 \\
\hline $6-F-33$ & $\mathrm{~T} 3$ & $\mathrm{~A}$ & 60 \\
\hline $7-\mathrm{M}-29$ & T5 & A & 55 \\
\hline $8-\mathrm{M}-32$ & $\mathrm{C} 8$ & $\mathrm{~B}$ & 53 \\
\hline $9-\mathrm{M}-40$ & $\mathrm{C} 7$ & B & 50 \\
\hline $10-\mathrm{F}-35$ & T9 & B & 46 \\
\hline $11-\mathrm{M}-38$ & $\mathrm{~T} 4$ & $\mathrm{~B}$ & 53 \\
\hline $12-\mathrm{M}-31$ & T5 & $\mathrm{A}$ & 54 \\
\hline \multicolumn{4}{|c|}{ Resiniferatoxin group } \\
\hline $1-M-30$ & T5 & $\mathrm{A}$ & 53 \\
\hline $2-F-38$ & $\mathrm{~T} 4$ & $\mathrm{~A}$ & 49 \\
\hline $3-\mathrm{M}-33$ & $\mathrm{~T} 10$ & B & 46 \\
\hline $4-F-28$ & T5 & A & 45 \\
\hline $5-\mathrm{M}-39$ & $\mathrm{~T} 10$ & B & 57 \\
\hline $6-\mathrm{M}-42$ & C5 & $\mathrm{B}$ & 52 \\
\hline $7-F-39$ & $\mathrm{~T} 1$ & $\mathrm{~B}$ & 45 \\
\hline $8-\mathrm{M}-34$ & $\mathrm{~T} 7$ & B & 48 \\
\hline $9-\mathrm{M}-38$ & T11 & B & 47 \\
\hline $10-\mathrm{M}-35$ & $\mathrm{C} 6$ & B & 55 \\
\hline $11-F-40$ & $\mathrm{~T} 10$ & B & 48 \\
\hline $12-\mathrm{M}-37$ & $\mathrm{~T} 6$ & B & 52 \\
\hline
\end{tabular}

tynin orally 3 times daily throughout the study. Any urinary tract infections were treated and patients were free of infection during the study.

Study plan. From the large pool of patients at our institutions 24 fulfilling all criteria were selected and block randomed by commercially available software to 2 groups of equal numbers (table 1). The synthetic capsaicin analogue N-vanillylnonanamide (Sigma Chemical Co.) and resiniferatoxin were dissolved in absolute ethanol and $0.9 \%$ sodium chloride solution, respectively, sterile filtered and divided into aliquots, which were stored at $-80 \mathrm{C}$. On the day of use instillations were prepared by diluting the stock solutions with $0.9 \%$ sodium chloride to the requisite concentrations. The dose of $2 \mathrm{mM}$. capsaicin was selected because it is the maximum concentration in ethanol reported in several clinical trials. ${ }^{2}$ The dose of $100 \mathrm{nM}$. resiniferatoxin was selected on the less secure basis that at least 1 group achieved good results at this concentration ${ }^{6}$ and another may have overachieved with $10 \mu \mathrm{M}$. resiniferatoxin. ${ }^{7}$

The 12 patients assigned to the capsaicin arm received intravesical instillation of $40 \mathrm{ml}$. $2 \%$ lidocaine, which remained in place for 20 minutes and was then drained. Subsequently $100 \mathrm{ml}$. capsaicin ( $2 \mathrm{mM}$. dissolved in $30 \%$ ethanol in $0.9 \%$ sodium chloride) were instilled via a $6 \mathrm{Fr}$ double lumen catheter with 1 lumen used to fill the bladder and the other used to measure intravesical pressure. The flow rate was $20 \mathrm{ml}$. per minute. The 12 patients assigned to the resiniferatoxin arm received an intravesical instillation of $100 \mathrm{ml}$. $0.9 \%$ sodium chloride solution containing $100 \mathrm{nM}$. resiniferatoxin at a flow rate of $20 \mathrm{ml}$. per minute. In each arm the solutions were retained in the bladder for 40 minutes with accompanying urodynamics (real-time cystometrography) while the drugs were indwelling.

Baseline urodynamic studies were duplicated 30 and 60 days after treatment. All patients maintained a voiding diary for 30 days before and throughout the 60-day study $(3 \times 30$ days of data). They recorded the number of daily catheterizations and episodes of incontinence. Local or systemic side effects were also noted. At the end of each study period patients underwent imaging assessment of the urinary tract by ultrasound and cystourethrography as indicated.

Data analysis. Descriptive values are expressed as the mean plus or minus standard deviation. Intergroup differ- ences were assessed by Student's t test for independent measures. Comparisons of data were assessed by Student's t test for dependent measures. Comparisons of frequencies were performed using the chi-square test with differences considered significant at $\mathrm{p}<0.05$.

\section{RESULTS}

Preliminary assessment. Initially 14 males and 6 females had incontinence episodes and used pads or external collecting devices, while the remaining 4 were dry (table 2 ). There were no significant differences in patients who received resiniferatoxin and capsaicin in regard to the frequency of catheterization, daily incontinent episodes or the number using pads and/or external devices. On baseline urodynamics all patients showed detrusor hyperreflexia, including 15 with detrusor sphincter dyssynergia, decreased bladder capacity and urinary leakage. None had consistently decreased compliance to less than $20 \mathrm{ml} . / \mathrm{cm}$. water. There was no significant difference in maximum bladder capacity, uninhibited detrusor contraction threshold or maximum amplitude in patients assigned to the capsaicin or resiniferatoxin arm (table 3).

Urodynamic monitoring during intravesical instillation. For resiniferatoxin 11 of the 12 patients completed the instillation without phasic detrusor contractions or urinary leakage. Treatment was aborted in 1 patient because of marked uninhibited detrusor contractions. There were no local or systemic side effects.

For capsaicin 5 of the 12 patients had autonomic dysreflexia with headache, anxiety, nausea, sweating, piloerection, bradycardia and blood pressures elevated to a mean of $172 /$ $102 \mathrm{~mm}$. Hg. The bladder was evacuated in 4 patients at 24, 31,35 and 38 minutes, respectively, because of spasms and leakage, while another received nifedipine sublingually at 35 minutes. The condition resolved rapidly in all 5 cases. Most patients reported increased spasticity and leg spasms, and 6 with incomplete lesions had suprapubic discomfort. These side effects, including minor hematuria in 5 patients, resolved within 4 to 11 days.

At 30-day followup (tables 2 and 3). In patients who received resiniferatoxin mean catheterization decreased from $6.6 \pm 0.7$ to $4.9 \pm 1.0(\mathrm{p}<0.001)$ and mean incontinence episodes decreased from $4.2 \pm 2.3$ to $1.7 \pm 2.5(\mathrm{p}<0.05)$. Seven patients were completely dry, clinical improvement was satisfactory in 4 and 1 in whom treatment was aborted failed to benefit. Urodynamic evaluation showed a significant increase in the uninhibited detrusor contraction threshold and in maximum bladder capacity but no modification in uninhibited detrusor contraction amplitude or compliance.

Overall patients assigned to the capsaicin arm did not have significant changes in the frequency of catheterization or incontinent episodes. There was a nonsignificant increase in the mean uninhibited detrusor contraction from $165 \pm 47$ to

TABle 2. Patients using pads or devices, and catheterization and incontinence episodes daily before, and 30 and 60 days after treatment

\begin{tabular}{lccc}
\hline & Pretreatment & 30 Days & 60 Days \\
\hline No. using pads or devices: & & & \\
$\quad$ Resiniferatoxin & 10 & $5^{*}$ & $5^{*}$ \\
$\quad$ Capsaicin & 10 & 9 & 9 \\
Mean No. catheteriza- & & & \\
$\quad$ tions daily \pm SD: & $6.6 \pm 0.7$ & $4.9 \pm 1.0 \ddagger$ & $4.8 \pm 0.8 \dagger$ \\
$\quad$ Resiniferatoxin & $5.8 \pm 1.1$ & $5.6 \pm 0.9$ & $5.6 \pm 1.0$ \\
$\quad$ Capsaicin & & & \\
Mean No. incontinence & & & \\
$\quad$ episodes daily \pm SD: & $4.2 \pm 2.3$ & $1.7 \pm 2.5^{*}$ & $1.9 \pm 2.3^{*}$ \\
$\quad$ Resiniferatoxin & $3.0 \pm 2.1$ & $2.0 \pm 2.0$ & $2.3 \pm 1.9$ \\
$\quad$ Capsaicin & & & \\
\hline
\end{tabular}

* Versus pretreatment $\mathrm{p}<0.05$.

$\dagger$ Versus pretreatment $\mathrm{p}<0.001$ 
TABLE 3. Urodynamic studies of uninhibited detrusor contraction, bladder capacity and compliance before, and 30 and 60 days after treatment

\begin{tabular}{|c|c|c|c|}
\hline & Pretreatment & 30 Days & 60 Days \\
\hline \multicolumn{4}{|c|}{ Mean uninhibited detrusor contraction threshold $\pm \mathrm{SD}$ (ml.): } \\
\hline Resiniferatoxin & $176 \pm 54$ & $250 \pm 107^{*}$ & $275 \pm 98 \dagger$ \\
\hline Capsaicin & $165 \pm 47$ & $199 \pm 58$ & $195 \pm 64$ \\
\hline \multicolumn{4}{|c|}{ Mean uninhibited detrusor contraction max. amplitude $\pm \mathrm{SD}$ (cm. water): } \\
\hline Resiniferatoxin & $71 \pm 28$ & $71 \pm 36$ & $68 \pm 26$ \\
\hline Capsaicin & $76 \pm 15$ & $70 \pm 21$ & $69 \pm 27$ \\
\hline \multicolumn{4}{|c|}{ Mean max. bladder capacity $\pm \mathrm{SD}(\mathrm{ml}$.$) :$} \\
\hline Resiniferatoxin & $196 \pm 75$ & $365 \pm 113 \ddagger$ & $357 \pm 101 \ddagger$ \\
\hline Capsaicin & $183 \pm 59$ & $219 \pm 94$ & $215 \pm 74$ \\
\hline \multicolumn{4}{|c|}{ Mean bladder compliance $\pm \mathrm{SD}(\mathrm{ml} . / \mathrm{cm}$. water $)$ : } \\
\hline Resiniferatoxin & $35 \pm 10$ & $35.7 \pm 8.6$ & $33.9 \pm 6.3$ \\
\hline Capsaicin & $37 \pm 12.5$ & $36.3 \pm 8.5$ & $37.8 \pm 9.1$ \\
\hline
\end{tabular}

* Versus pretreatment $\mathrm{p}<0.05$.

+ Versus pretreatment $\mathrm{p}<0.01$

$\ddagger$ Versus pretreatment $\mathrm{p}<0.001$.

$199 \pm 58 \mathrm{ml}$. and in mean bladder capacity from $183 \pm 59$ to $219 \pm 94 \mathrm{ml}$. No local or systemic side effects were noted at this point.

At 60-day followup (tables 2 and 3). Patients in the resiniferatoxin group substantially maintained the clinical and urodynamic benefits observed at the previous followup. The mean frequency of catheterization and of incontinent episodes was not significantly different than at the 30-day assessment. Complete continence was maintained in 7 patients $(58.3 \%)$, clinical improvement persisted in $4(33.3 \%)$ and treatment failed in 1 .

The 12 patients in the capsaicin group maintained the slight increase in the uninhibited detrusor contraction threshold and in cystometric capacity previously achieved. However, patients 3, 5, 7 and 11 (33\%) achieved more substantial clinical and urodynamic improvement, which narrowly failed to reach significance ( $p=0.08$ to 0.052). Compared with pretreatment levels the mean number of incontinent episodes decreased from 4.5 to 1.5 daily, the mean uninhibited detrusor contraction threshold increased from 173 to $240 \mathrm{ml}$. and mean maximum bladder capacity increased from 208 to $285 \mathrm{ml}$. In addition, there was no modification in detrusor-sphincter dyssynergia in any of the 15 patients with that condition.

\section{DISCUSSION}

Electrophysiological and pharmacological studies in animals demonstrate that interruption of the spinal cord pathways produces considerable reorganization of the micturition reflex and C-fiber bladder afferents become mechanoreceptive and initiate reflex voiding. ${ }^{8}$ Systemic administration of capsaicin decreases the number of bladder C-fiber afferents and suppresses hyperreflexic activity in chronic spinal cord injured cats. ${ }^{8}$ An increasing number of experimental clinical studies were designed to investigate these effects in humans with detrusor hyperreflexia and results after intravesical capsaicin were generally encouraging, in that 6 detailed clinical benefits in $40 \%$ to $100 \%$ of patients and urodynamic improvement in $60 \%$ to $100 \% .^{2}$ In contrast, only 4 of our 12 patients (33\%) improved clinically and urodynamically after capsaicin treatment. These inferior results require further analysis.

Capsaicin administration was preceded by intravesical lidocaine. ${ }^{9}$ Does lidocaine attenuate the potential benefits of capsaicin? We consider this hypothesis most unlikely because capsaicin causes excitation (nociception) and then desensitization through nonselective receptor mediated ion channels, whereas lidocaine blocks axonal transmission through voltage dependent sodium channels. In animal studies the local anesthetics lidocaine (amide type) and tetracaine (ester type) partially prevented the excitatory effects of capsaicin ${ }^{10}$ and resiniferatoxin, ${ }^{11}$ respec- tively, without influencing desensitization. Also, in clinical studies pretreatment with lidocaine did not decrease capsaicin efficacy ${ }^{12,13}$ and Dasgupta et al noted an improved response to capsaicin after accelerated administration of intravesical lidocaine because of fewer problems during treatment. ${ }^{14}$

An overview of clinical studies of capsaicin does little to clarify the situation. Studies of at least 9 distinct diseases or traumas, of which the most common is multiple sclerosis, can be further subdivided into complete or incomplete lesions of the spinal cord at all levels. Some groups administered $1 \mathrm{mM}$. solutions, ${ }^{15-17}$ others gave $2 \mathrm{mM} .{ }^{18}$ and yet others used the 2 concentrations in the same groups of patients. ${ }^{9,12,13}$ Vaidyanathan et al examined vesical nerve fiber patterns and noted marked variation in patients with the same disease or trauma of the spinal cord at similar levels, ${ }^{19}$ and so individual characteristics of bladder nerve involvement may have a role. Disease duration of an average of 52 months in our patients may affect the response and yet it is rarely reported. de Seze et al reported disease duration but the majority of their patients had multiple sclerosis. ${ }^{16}$ Simply stated too many independent variables are involved to make general comparisons. Specifically the only comparable investigations were those of Geirsson et al.18 Using $2 \mathrm{mM}$. capsaicin they achieved urodynamic improvement in 9 of 10 spinal cord injured patients versus our 4 of 12 and yet only 4 of 10 had a clinical response versus our 4 of 12 .

Resiniferatoxin with a molecular weight of approximately $630 \mathrm{Da}$. contains the same homovanillyl group determining biological activity as capsaicin with a molecular weight of approximately $300 \mathrm{Da}$. and it is appealing because there is minimal to absent acute toxicity. ${ }^{6,7}$ The current study demonstrates that resiniferatoxin, even at $1 / 20,000$ the concentration of capsaicin, was more effective and safer than capsaicin. Two previous studies that were neither prospective nor randomized also indicate a superior response to resiniferatoxin. ${ }^{7,20}$ In common with other researchers we are confronted by an apparent paradox. Capsaicin and resiniferatoxin interact with the same specific recognition site (vanilloid receptor subtype I) and yet equipotent and less than equipotent (95\% less in our study) concentrations of resiniferatoxin resulted in generally superior therapeutic effects with few if any of the noxious side effects associated with capsaicin. This pharmacological anomaly has been ascribed somewhat hesitantly to low concentrations of resiniferatoxin desensitizing the afferent neurons without inducing an initial sensitization-excitation phase, as with capsaicin. ${ }^{2}$ The overall situation remains unclear because, although techniques for the intravesical administration of capsaicin are now reasonably standardized, the same cannot be said for resiniferatoxin instillations since concentrations of 1 to 10 
$\mu \mathrm{M}$., volumes of 30 to $100 \mathrm{ml}$. with and without $10 \%$ ethanol, and a wide array of therapeutic schedules have been reported. ${ }^{2,6,7}$ No mass balance studies are available to indicate the proportional uptake of the 2 vanilloids and, therefore, to our knowledge the important role of urothelial permeability remains unknown. However, it is possible to outline some qualitative pharmacokinetics of these agents administered intravesically.

Resiniferatoxin is slightly soluble in water since $1 \mathrm{mM}$. aqueous solutions do not precipitate and ethanol is not a chemical necessity for intravesical instillation. When solubilized in water alone, the size of the resiniferatoxin molecule precludes intracellular penetration of urothelial cells and to reach $\mathrm{C}$-fibers resiniferatoxin must pass through the intercellular channels (pores) associated with the junctional complex between the lateral cell membranes. ${ }^{21}$ When in the region of the filamentous lamina propria, diffusion to receptor sites on C-fibers should be rapid. For capsaicin the situation is more complicated. For all practical purposes it is insoluble in water and to attain the desired concentrations of 1 to $2 \mathrm{mM}$. $30 \%$ ethanol is most commonly used. When instilled as a control, $30 \%$ ethanol causes side effects, namely bladder inflammation, that are indistinguishable from side effects caused by ethanol with capsaicin, while onset is rapid ("immediate") and the adverse effects persist for 1 to 2 weeks. ${ }^{16}$ The luminal plasma membrane of the urothelial cells is no barrier to the membrane fluidizing effects of ethanol, ${ }^{22}$ which moves intracellularly, entraining its solubilized capsaicin. Alcohol induced tissue damage ensues, followed by the inflammatory cascade, including mediators of inflammation that induce the excitation of capsaicin sensitive neurons. ${ }^{2}$ Therefore, ethanol diverts a proportion of capsaicin to where it cannot exert any pharmacological effects and is also responsible for some if not most of the noxious side effects. In this context it is worth noting that intravesical $10 \%$ ethanol with resiniferatoxin caused some vague temporary discomfort, ${ }^{6}$ a solution of ethanol $90 \%$ has caused permanent bladder damage dand $^{23}$ and. capsaicin with polysorbate (Tween) as the vehicle caused no major side effects. ${ }^{24}$

Nothing in this commentary is at variance with concepts involving resiniferatoxin and capsaicin actions on different C-fiber subsets ${ }^{25}$ or vanilloid receptor subtypes. ${ }^{26}$ Rather it emphasizes the fact that ethanol concentrations should be restricted to the necessary minimum when this agent is given as an intravesical vehicle. Capsaicin (1 mM.) in 15\% ethanol with $85 \mathrm{ml}$. saline is chemically feasible and yet the majority of clinical reports describe $30 \%$ ethanol as the vehicle for $1 \mathrm{mM}$. capsaicin. ${ }^{9,12,13,15-17}$ With hindsight it is obvious that this situation causes problems when assessing the relative merits of 1 and $2 \mathrm{mM}$. solutions, and weighs against the lower concentration because of the unnecessarily severe inflammatory effects that presumably occur. A prospective study of $1 \mathrm{mM}$. capsaicin in $15 \%$ and $2 \mathrm{mM}$. in $30 \%$ ethanol should further clarify and define the role of intravesical capsaicin for treating detrusor hyperreflexia.

\section{CONCLUSIONS}

In spinal cord injured patients with detrusor hyperreflexia refractory to oral oxybutynin therapy intravesical resiniferatoxin provided superior clinical and urodynamic benefits, and had fewer side effects than intravesical capsaicin, of which the potential benefits were probably decreased by premature evacuation of the drug in a third. Due to its minimal to absent toxicity we would recommend $100 \mathrm{ml}$. intravesical resiniferatoxin (100 nM.) in $0.9 \%$ sodium chloride in cases refractory to standard therapy with few restrictive criteria.

\section{REFERENCES}

1. Kim, D. Y. and Chancellor, M. B.: Intravesical neuromodulatory drugs: capsaicin and resiniferatoxin to treat the overactive bladder. J Endourol, 14: 97, 2000
2. Chancellor, M. B. and de Groat, W. C.: Intravesical capsaicin and resiniferatoxin therapy: spicing up the ways to treat the overactive bladder. J Urol, 162: 3, 1999

3. Avelino, A., Cruz, F. and Coimbra, A.: Intravesical resiniferatoxin desensitizes rat bladder sensory fibres without causing intense noxious excitation. A c-fos study. Eur J Pharmacol, 378: 17, 1999

4. Ditunno, J. F., Young, W., Donovan, W. H. et al: The international standards booklet for neurological and functional classification of spinal cord injury: American Spinal Injury Association. Paraplegia, 32: 70, 1994

5. Stöhrer, M., Goepel, M., Kondo, A. et al: The standardization of terminology in neurogenic lower urinary tract dysfunction with suggestion for diagnostic procedures: International Continence Society Standardization Committee. Neurourol Urodyn, 18: 139, 1999

6. Silva, C., Rio, M. E. and Cruz, F.: Desensitization of bladder sensory fibers by intravesical resiniferatoxin, a capsaicin analog: long-term results for the treatment of detrusor hyperreflexia. Eur Urol, 38: 444, 2000

7. Lazzeri, M., Spinelli, M., Beneforti, P. et al: Intravesical resiniferatoxin for the treatment of detrusor hyperreflexia refractory to capsaicin in patients with chronic spinal cord disease. Scand J Urol Nephrol, 32: 331, 1998

8. Yoshimura, N.: Bladder afferent pathway and spinal cord injury: possible mechanisms inducing hyperreflexia of the urinary bladder. Prog Neurobiol, 57: 583, 1999

9. Fowler, C. J., Beck, R. O., Gerrards, S. et al: Intravesical capsaicin for treatment of detrusor hyperreflexia. J Neurol Neurosurg Psychiatry, 57: 169, 1994

10. Avelino, A., Cruz, F. and Coimbra, A.: Lidocaine prevents noxious excitation of bladder afferents induced by intravesical capsaicin without interfering with the ensuing sensory desensitization: an experimental study in the rat. J Urol, 159: 567, 1998

11. Craft, R. M. and Porreca, F.: Tetracaine attenuates irritancy without attenuating desensitization produced by intravesical resiniferatoxin in the rat. Pain, 57: 351, 1994

12. Chandiramani, V. A., Peterson, T., Duthie, G. S. et al: Urodynamic changes during therapeutic intravesical instillations of capsaicin. Br J Urol, 77: 792, 1996

13. De Ridder, D., Chandiramani, V., Dasgupta, P. et al: Intravesical capsaicin as a treatment for refractory detrusor hyperreflexia: a dual center study with long term followup. J Urol, 158: 2087, 1997

14. Dasgupta, P., Fowler, C. J. and Stephen, R. L.: Electromotive drug administration of lidocaine to anesthetize the bladder before intravesical capsaicin. J Urol, 159: 1857, 1998

15. Cruz, F., Guimarães, M., Silva, C. et al: Desensitization of bladder sensory fibers by intravesical capsaicin has long lasting clinical and urodynamic effects in patients with hyperactive or hypersensitive bladder dysfunction. J Urol, 157: 585,1997

16. de Seze, M., Wiart, L., Joseph, P. A. et al: Capsaicin and neurogenic detrusor hyperreflexia. A double-blind placebocontrolled study in 20 patients with spinal cord lesions. Neurourol Urodyn, 17: 513, 1998

17. Petersen, T., Nielsen, J. B. and Schrøder, H. D.: Intravesical capsaicin in patients with detrusor hyper-reflexia. A placebocontrolled cross-over study. Scand J Urol Nephrol, 33: 104, 1999

18. Geirsson, G., Fall, M. and Sullivan, L.: Clinical and urodynamic effects of intravesical capsaicin treatment in patients with chronic traumatic spinal detrusor hyperreflexia. J Urol, 154: 1825,1995

19. Vaidyanathan, S., van Velzen, D., Parsons, K. F. et al: Nerve fibres in urothelium and submucosa of neuropathic urinary bladder: an immunohistochemical study with S-100 and neurofilament. Paraplegia, 34: 137, 1996

20. Park, W. H., Kim, H. G., Park, B. J. et al: Comparison of the effects of intravesical capsaicin and resiniferatoxin for treatment of detrusor hyperreflexia in patients with spinal cord injury. Neurourol Urodyn, 18: 402, 1999

21. Mishina, T., Watanabe, H., Kobayashi, T. et al: Absorption of anticancer drugs through bladder epithelium. Urology, 27: 148, 1986

22. Chin, J. H. and Goldstein, D. B.: Membrane: disordering action of ethanol. Mol Pharmacol, 19: 425, 1981 
23. Christmas, T. J., Chapple, C. R., Payne, S. D. et al: Bonney's blue cystitis: a warning. Br J Urol, 63: 281, 1989

24. Lai, R. S. and Hassouna, M. M.: Intravesical capsaicin therapy produces a dose dependent reduction of detrusor leak point pressure in spinal cord injury patients. J Urol, part 2, 163: 245 , abstract 1090, 2000

25. Sengupta, J. N. and Gebhart, G. F.: Mechanosensitive afferent fibers in the gastrointestinal and lower urinary tracts. In: Visceral Pain Progress in Pain Research and Management. Edited by G. F. Gebhart. Seattle: IASP Press, vol. 5, pp. 75-98, 1995

26. Acs, G., Biro, T., Acs, P. et al: Differential activation and desensitization of sensory neurons by resiniferatoxin. J Neurosci, 17: 5622,1997 